\title{
An All-Payer Risk Model for Super-Utilization in a Large Safety Net System
}

\author{
Jeremy Ziring, $B A^{7}$, Spriha Gogia, $P h D, M P H^{2}$, Remle Newton-Dame, $M P H^{2}$, Jesse Singer, $D O, \mathrm{MPH}^{2}$, \\ and Dave A. Chokshi, MD, MSc $c^{1,2}$
}

${ }^{1}$ NYU School of Medicine, New York, NY, USA; ${ }^{2}$ NYC Health + Hospitals, New York, NY, USA.

$\mathrm{J}$ Gen Intern Med 33(5):596-8

DOI: $10.1007 / \mathrm{s} 11606-018-4346-5$

(c) The Author(s) 2018. This article is an open access publication

\section{INTRODUCTION}

Identifying patients at high risk for super-utilization of inpatient and emergency services - and proactively managing their care - are key strategies for healthcare systems aiming to improve population health and control costs. Traditional claims-based risk scores are inadequate for uninsured patients and patients with insurance churn, and many safety net systems do not have an electronic health record (EHR) capable of advanced analytics.

As the largest safety net system in the country, NYC Health + Hospitals serves a high-need population, including thousands of patients with multiple, interlinked medical, behavioral health, and social issues. More than half of the system's patients had an emergency room (ER) visit in the past year. Seventeen percent had two or more visits, and 250 patients averaged at least a day a week in one of our emergency rooms. NYC Health + Hospitals also provides half of all uninsured emergency and inpatient care for New Yorkers, including more than $80 \%$ of uninsured non-emergency services. ${ }^{1}$ To be successful, risk prediction strategies must encompass NYC Health + Hospitals' entire patient population.

\section{METHODS}

Our objective was to develop a payer-agnostic risk model for super-utilization using administrative and clinical data from the largest safety net system in the USA. We selected adults that visited an NYC Health + Hospitals acute care, community health center, or skilled nursing facility in 2014 (index year) and were not designated pregnant or actively incarcerated during the study period. Patients were randomly assigned to a development (80\%) or validation (20\%) cohort, using SAS Enterprise Guide 7.11 (SAS Institute). Internal administrative data provided utilization, demographic and scheduling data, and diagnoses came from clinical data. Our primary outcome was super-utilization within our system during 2015 (prediction year), defined as $\geq 10$ days in inpatient care or the emergency room. To identify candidate variables, we aligned available data elements to key factors in the literature and clinical

Published online February 20, 2018 guidelines. ${ }^{2}$ Stepwise selection identified the final logistic model and generated algorithmic weights; model discrimination was assessed using the c-statistic. Our large sample size precluded the Hosmer-Lemeshow test. ${ }^{3}$ We converted weights to a risk algorithm using methods described by Sullivan et al. ${ }^{4}$ Risk score performance was assessed on the validation set using positive predictive value for 2015 super-utilization among the top $1 \%$ of 2014 patients (high risk). Figure was generated using Tableau 10.2 (Tableau Software).

\section{RESULTS}

We retained 643,475 NYC Health + Hospitals patients in the development cohort, of which $45.8 \%$ were males, $33.9 \%$ were enrolled in Medicaid at their most recent visit in 2014, and

Table 1 Payer-Agnostic Risk Score from a 2014/2015 Safety Net Cohort

\begin{tabular}{lll}
\hline \hline Variable & Value & Risk points \\
\hline ED visits in 2014 & 1-2 visits & 2.4 \\
& 3-4 visits & 5.7 \\
Inpatient visits in 2014 & 1 visits & 10.3 \\
& 2 visits & 6.7 \\
& 3 visits & 8.2 \\
4+ visits & 9.1 \\
in inpatient/ED days & & 11.3 \\
Marital status category & Single, never married & 3.9 \\
& Separated, widowed, & 1.9 \\
Gender & and divorced & 3.1 \\
Age category & Male & 1.7 \\
& 45-64 & 2.6 \\
Diagnosis flags & 65-80 & 6.1 \\
& 281 & 9.4 \\
& Alcohol disorders & 4.2 \\
& Schizophrenia & 7.7 \\
& Mood disorders & 1.7 \\
& Heart disease & 1.4 \\
& Substance disorders & 2.6 \\
medicine clinic visits & Chronic kidney disease & 5.6 \\
Payer changes & Asthma & 1.7 \\
& Diabetes & 1.1 \\
& Sickle cell & 5.6 \\
& & 1.0 \\
& 1-2 zip changes & 1.8 \\
& 3+ zip changes & 2.3 \\
1-2 payer changes & 1.0 \\
& 3-4 payer changes & 1.4 \\
5+ payer changes & 2.0 \\
\hline
\end{tabular}




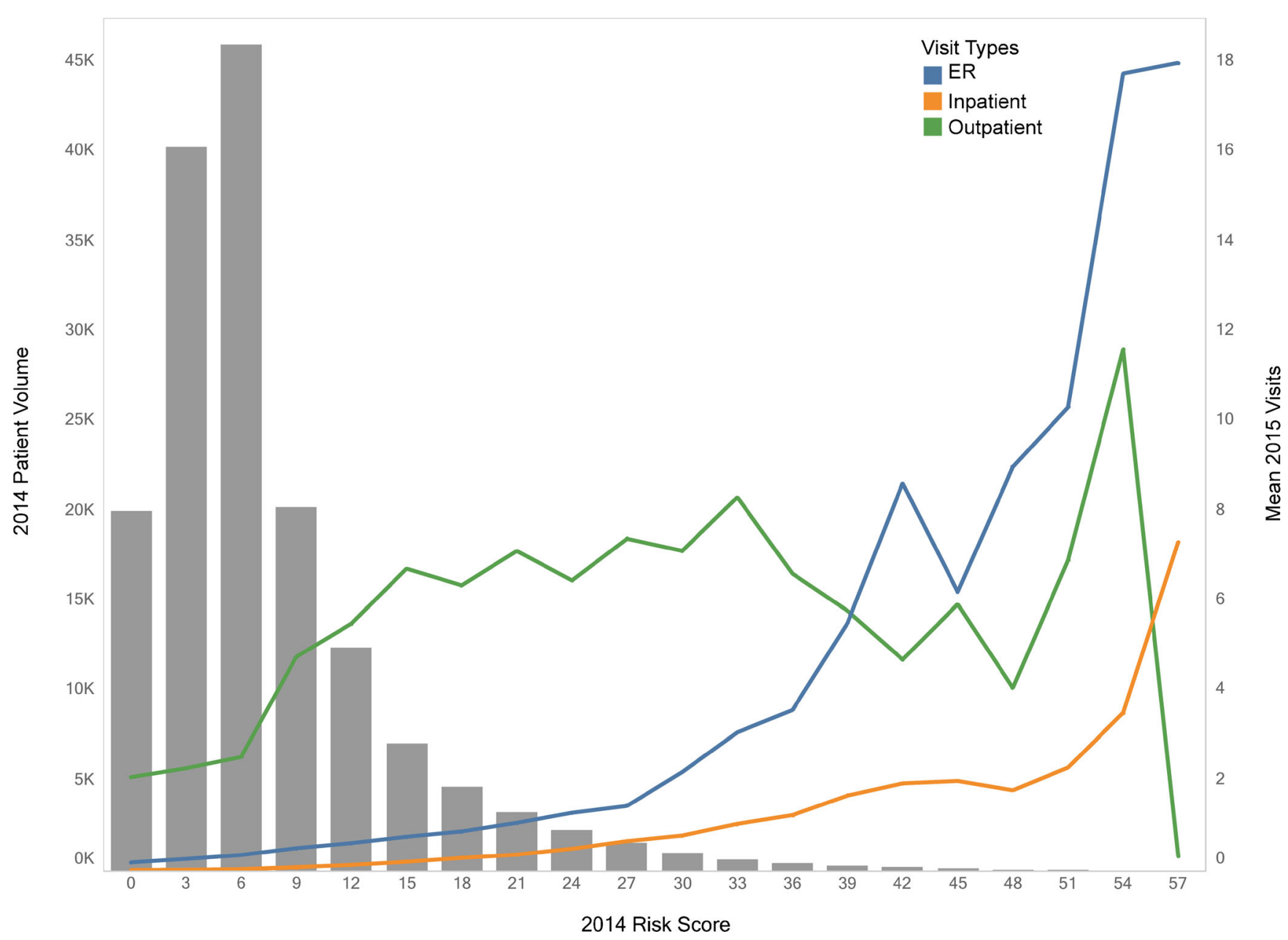

Fig. 1 Mean 2015 emergency, inpatient, and outpatient utilization vs 2014 risk score distribution among NYC Health + Hospitals patients.

$37.4 \%$ were uninsured. Median age was 45 . In 2014, $4.8 \%$ of this cohort was super-utilizers, dropping to $2.9 \%$ in 2015.

In the final algorithm, the strongest predictors of superutilization were inpatient and ER visits; older age; and diagnoses of schizophrenia, chronic kidney disease, and sickle cell disease (Table 1). We did not retain race, which was significant, due to data validity concerns. The final model's c-statistic was 0.86 , outperforming established readmission models tested at other urban public hospitals and falling above the 0.70 threshold of acceptable discrimination. ${ }^{5}$

In the validation set $(n=160,868)$, the model identified 2015 super-utilization among 2014 high risk patients with a $44.8 \%$ positive predictive value. In 2015 , high-risk patients had an average of 1.5 inpatient and 5.4 ER visits, and 75.5\% had $\geq 1 \mathrm{ER} /$ inpatient visit. As risk of super-utilization increased, average ER/inpatient visits rose while outpatient visits leveled off and then declined (Fig. 1).

\section{DISCUSSION}

Readily available clinical and administrative data successfully discriminated risk of future super-utilization for both uninsured and insured patients across all payers at a large, urban safety net hospital system. Proxies such as zip code changes, payer flux, and missed clinic visits helped represent poorly documented social determinants of health. This model did not require advanced EHR functionality or proprietary claim-based rules, making it timely and affordable for our system. A payeragnostic approach to risk scoring may increase clinician buyin, since it covers the provider's full panel of patients and improves targeting of resource-intensive interventions. We hope that this algorithm helps initiate thoughtful, populationtargeted risk stratification strategies at other delivery systems serving vulnerable patients.

Acknowledgments: We are grateful to NYC Health + Hospitals' Accountable Care Organization for providing us with their risk scoring approach to Medicare claims data, which informed development of our algorithm.

Corresponding Author: Dave A. Chokshi, MD, MSc; NYC Health + Hospitals, New York, NY, USA (e-mail: dave.chokshi@nychhc.org).

\section{Compliance with Ethical Standards:}

Conflicts of Interest: The authors declare that they do not have a conflict of interest. 
Open Access This article is distributed under the terms of the Creative Commons Attribution 4.0 International License (http:// creativecommons.org/licenses/by/4.0/), which permits unrestricted use, distribution, and reproduction in any medium, provided you give appropriate credit to the original author(s) and the source, provide a link to the Creative Commons license, and indicate if changes were made.

\section{REFERENCES}

1. The City of New York. One New York-health care for our neighborhoods: Transforming health + hospitals. New York: The City of New York; 2016. Available from http://wwwl.nyc.gov/assets/home/downloads/pdf/reports/2016/Health-and-Hospitals-Report.pdf.
2. Billings J, Georghiou T, Blunt I, Bardsley $\mathbf{M}$. Choosing a model to predict hospital admission: an observational study of new variants of predictive models for case finding. BMJ Open 2013;3(8):e003352.

3. Kramer AA, Zimmerman JE. Assessing the calibration of mortality benchmarks in critical care: The Hosmer-Lemeshow test revisited. Crit Care Med 2007:35(9):2052-2056.

4. Sullivan LM, Massaro, J. M. and D'Agostino, R. B. Presentation of multivariate data for clinical use: The Framingham Study risk score functions. Stat Med 2004;23:1631-1660.

5. Amarasingham R, Velasco F, Xie B, Clark C, Ma $\mathbf{Y}$, Zhang S, et al. Electronic medical record-based multicondition models to predict the risk of 30 day readmission or death among adult medicine patients: validation and comparison to existing models. BMC Med Inform Decis Mak 2015; 15:39. 